\title{
A PRIMITIVE EXECUTION MODEL FOR HETEROGENEOUS MODELING
}

\author{
Frédéric Boulanger \\ Supélec - Département Informatique, \\ 3 rue Joliot-Curie, 91192 Gif-sur-Yvette cedex, France \\ Email: Frederic.Boulanger@supelec.fr \\ Guy Vidal-Naquet \\ Supélec and Université Paris-Sud, \\ 3 rue Joliot-Curie, 91192 Gif-sur-Yvette cedex, France \\ Email: Guy.Vidal-Naquet@supelec.fr
}

Keywords: Heterogeneous modeling, Models of computation, Execution models.

Abstract: Heterogeneous modeling is modeling using several modeling methods. Since many different modeling methods are used in different crafts, heterogeneous modeling is necessary to build a heterogeneous model of a system that takes the modeling habits of the designers into account.

A model of computation is a formal description of the behavioral aspect of a modeling method. It is the set of rules that allows to compute the behavior of a system by composing the behaviors of its components. Heterogeneous modeling allows parts of the system to obey some rules while other parts obey other rules for the composition of their behaviors.

Computing the behavior of a system which is modeled using several models of computation can be difficult if the meaning of each model of computation, and what happens at their boundary, is not well defined. We propose an execution model that provides a framework of primitive operations that allow to express how a model of computation is interpreted in order to compute the behavior of a model of a system. When models of computation are "implemented" in this execution model, it becomes possible to specify exactly what is the meaning of the joint use of several models of computation in the model of a system.

\section{CONTEXT}

The design of most complex systems appeals to different crafts that are organized around sets of specific design methods, e.g. for industrial control or signal processing. These methods are adapted to specific aspects of a craft, and designers have a correct intuition of their semantics.

When integrating the different parts of a system, we generally translate the model of each part into a common low level formalism, or even into a common implementation language. By doing so, we loose all the information that tells how we went from the specification of the subsystem to its model. Therefore, when building the whole system, we cannot take advantage of the different choices of realization offered by the model, since they have been "frozen" in the low level implementation.

Another issue is that, when validating the behavior of the whole system, it will be difficult to find what should be changed in the model of a subsystem to insure a global property, since the low level implementation does not carry enough information about the design of the subsystem.
Heterogeneous modeling tries to overcome these issues by allowing to describe the whole system as a composition of subsystems that are designed according to different methods (Liu et al., 2003).

It does not provide a greater expressive power than other modeling techniques, but it allows the different teams that work on the design of a system to share a common model of the system, while using their own modeling techniques.

In the following, we present the actor paradigm for heterogeneous modeling and we propose a constructive method for computing the behavior of heterogeneous models. Projects like KerMeta (Fleurey et al., 2006) or Rosetta (Kong and Alexander, 2003) are related to our works, but focus on different objectives: KerMeta defines behaviors for the elements of the Meta-Object Facility (MOF) of the OMG, while Rosetta defines the combination of models of computation and uses a hierarchy of compatible models of computation. Our objective is to provide a framework in which any model of computation may be used to compute the interactions of components of a system. In this paper, we focus on the steps that are required to compute the behavior of a model of a system. 


\section{ACTORS, PORTS, RELATIONS}

Our approach to modeling is based on the actor paradigm: a system is built from components named actors. Actors have properties and communicate through ports. Ports have properties and are linked by relations which also have properties. So, building a model of a system amounts to using some actors, to set their properties and the properties of their ports, and to build relations between the ports of the actors. The effective behavior of the model is obtained by interpreting the properties and the relations according to a model of computation.

Actors, ports, properties and relations are the elements of the abstract syntax of actor-oriented modeling. Models of computation are semantics for this abstract syntax: a model of computation is an interpretation of the relations between the ports of actors and the properties of these relations.

\subsection{Roles of a Model of Computation}

A model of computation allows to compute the observable behavior of a model of a system from the individual observable behaviors of its components. For instance, on figure 1 , the $\mathrm{MoC}_{e x t}$ model of computation computes the behavior of the top-level model that contains actors A and B. This model of computation is in charge of computing the status (availability of data and value) of $\mathrm{B}_{\text {in }}$ from the status of $\mathrm{A}_{\text {out }}$. The status of $\mathrm{A}_{\text {out }}$ is determined from the status of $\mathrm{A}_{\text {in }}$ by the behavior of $A$. In the example, this behavior is described by a model that contains three actors and is governed by the $\mathrm{MoC}_{\text {int }}$ model of computation.

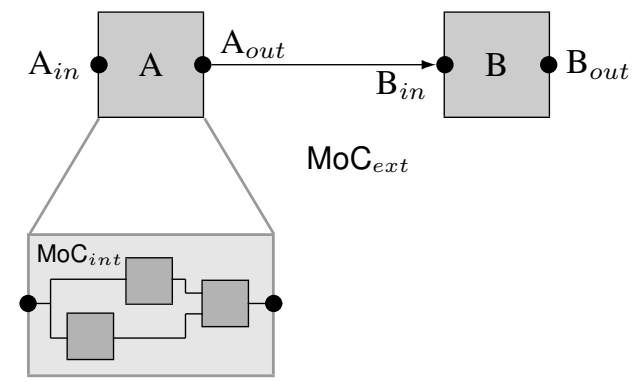

Figure 1: Internal and external models of computation

$\mathrm{MoC}_{\text {ext }}$ is the "external model of computation" of actor A. It is the model of computation that combines its behavior with the behavior of other actors to compute the behavior of a model. $\mathrm{MoC}_{i n t}$ is the "internal model of computation" of A. It is the model that computes the behavior of A by combining the behaviors of the actors of the model of A.

Information is obtained by observing the output ports of actors, so the model of computation must tell how information goes from output to input ports, and when it is available on input ports. We can consider the first aspect (the propagation of data from output ports to input ports) as communication, and the second aspect (when data is available) as control or synchronization.

Communication consists generally in copying data from output ports to input ports, but synchronization can be more complex because it defines the type of causality used by the model of computation. In some models of computation, data produced on an output port is available immediately on all input ports that are in relation with that output port. In other models of computation, a notion of "tick" is used to relax causality: a data sample produced on an output port is available on all inputs ports that are in relation with it, but only after the next tick. In other models of computation, a notion of time is introduced to label data samples with a time-stamp which tells when they are available.

Communication and synchronization aspects must be described precisely to define a model of computation. There are actually very few tools or languages for describing these aspects. Most of the time, one has to implement a model of computation in a generic programming language.

\section{EXECUTION MODEL}

When the precise semantics of a model of computation is coded in a programming language, it is generally done in the context of a framework like Ptolemy II (Brooks et al., 2005) that provides support for the abstract syntax of the models. For such a framework to support an open set of models of computation, it must consider components as black boxes that compute the availability and values of data on input ports from the availability and values of data on output ports.

We define here a generic execution model that can be used with any model of computation. It relies on models of computation for determining in which order the actors of a model should be observed, and how the values on input ports are computed from the values observed on output ports. This execution model has matured from previous works (Boulanger et al., 2004; Feredj et al., 2004) based on the Ptolemy framework.

\subsection{The nature of an execution}

Before defining our generic execution model, we must define what is an execution of a model of a system. In order to keep our approach generic, we ignore the internal mechanisms of an actor, to focus on what and 
when an actor produces data on its output ports, not how it produces it.

Therefore, in the following, we will never "trigger" the behavior of an actor, we will just observe its output ports. The behavior of an actor can occur any time - it can be a continuous process that runs during the whole execution of the model - but it must provide a coherent view each time its ports are observed. We use a stroboscopic effect to observe the actors of a model simultaneously in a series of snapshots. We consider that an execution of a model is a sequence of snapshots of the values available on the ports of the model. In a given snapshot, each port has a single defined value.

The nature of a snapshot depends on the model of computation. The execution model only tells the actors that a snapshot is going to be taken, and then asks them to approve the value of their ports as they appear on the snapshot. For an analogy, a photographer tells the actors "stay still", and then ask them if they are pleased by the picture.

This definition of an execution implies that we are only interested in discrete behaviors. This is because our goal is not to describe behaviors, but to compute them. For instance, when we consider a model of a physical system, like a system of ordinary differential equations, we are not interested in finding properties of these ODEs but in computing the value of the outputs of the system at a discrete set of instants.

Since we are interested in observations only, each model of computation is insulated from the definition of the others, and there is no need to define the composition of any pair of models of computation.

For instance, if an actor is a sensor which acquires information from the external world, what is interesting is the result of the measure, not the mechanism for elaborating this measure.

\subsection{Types of actors}

To define a generic execution model, we must consider the different ways with which an actor produces its outputs. Strict actors need to know the value of all their inputs to determine the value of all their outputs at once. With strict actors, a model cannot contain instantaneous causality loops because a strict actor cannot have an input that depends on the value of one of its outputs in the same snapshot. Non-strict actors can determine some of their outputs when they know the value of only some of their inputs. A delay is a non-strict actor: the value of its output depends only on its state, which in turn depends on the value that its input had in the previous snapshot. A logical OR gate is also a non-strict actor because its output is known as soon as one of its inputs is true.

When a model of computation supports non-strict actors, the values of the ports are determined itera- tively. First, actors are provided with known inputs and they determine part of their outputs. These newly determined outputs allow to compute new values for inputs according to the model of computation. These newly determined inputs allow actors to determine more outputs, and so on until all the ports have a known value. With such models of computation, it is necessary to tell actors when new inputs become available.

Actors, independently of their strict or non-strict nature, may not agree with the value they have computed for their outputs for the current snapshot. For instance, consider a level-crossing detector. It produces an event when a signal crosses a threshold. If the signal is computed by numerical integration of differential equations, the integration step is adjusted so that the value of the signal is computed with a given precision. However, the measure may be refused if the integration step is to large for the temporal precision required on the event, and the snapshot will be computed again with a smaller integration step. A snapshot is considered valid when all the actors of the model agree with the value assigned to their ports.

\subsection{Generic Execution Model}

The taxonomy of actors presented above, and the fact that we are only interested in observations of the ports of actors, not in the activity of the actors, allows us to define a generic execution model that is capable of executing models that obey any model of computation. To attain such universality, we made as few assumptions about actors as possible, and we rely on an operational description of the model of computation to schedule observations and to compute the value and availability of data on input ports from the data available on output ports.

One can wonder at the previous sentence since we are used to outputs computed from inputs, not the reverse. The key is to consider that if actors produce their outputs from their inputs, the model of computation interprets the relations between ports to determine what is available on inputs ports from what is available on output ports.

We can now describe the steps of our execution model to compute a snapshot of the execution of a model of a system, and define the primitive operations that an actor must provide:

1. the start_of_snapshot operation is invoked on each actor of the model. In response to this invocation, an actor prepares for the snapshot. For instance, an actor that acquires information from the environment of the system (reading data from a file, sampling a sensor) should do it during this step. 
2. the reset operation is invoked on each actor of the model. In response to this invocation, an actor resets its ports to the "unknown" state.

3. the update operation is invoked on the actors of the model returned by the schedule operation of the model of computation. In response to this invocation, an actor makes data available on its output ports. If the actor is strict, it makes data available on all its output ports. If it is non-strict, it makes data available only on the ports it can determine.

4. the operational description of the model of computation is used to compute the status (availability of data and value of the data) of the input ports from the status of the output ports.

5. if the model of computation determines that the snapshot is complete, go to step 6, else, go back to step 3. Steps 3 to 5 constitute an observation of the model.

6. the validate_snapshot operation is invoked on each actor of the model. In response to this operation, an actor considers the data available on its ports as definitive for this snapshot and tells whether it considers it as correct or not. If it does not validate the data, it should change some property of the model of computation (e.g. the integration step in our example with the level-crossing detector) so that a new computation of the snapshot will compute data that it may validate.

7. if all the actors of the model have validated the snapshot, go to step 8, else go back to step 2 to compute the snapshot again with the new parameters of the model of computation that have been set by the actors which have not validated the snapshot.

8. when all the actors of the model have validated the snapshot, the end_of_snapshot operation is invoked on each actor of the model. This operation tells actors that the snapshot is valid and that they can use the data available on their ports in their own activity or to update their internal state if any. Actors that provide data to the environment of the system (writing data to a file, driving an actuator) should do so during this step.

An actor should not update its internal state, change its activity or perform any operation that may have side effects on the environment between the start_of_snapshot and end_of_snapshot operations. The fact that a snapshot may be computed several times to converge toward a result that is accepted by all the actors must not be visible outside the model. For instance, if we consider our example of a level-crossing detector, it may be necessary to compute a snapshot several times before the integration step becomes small enough, but outside the model, only the last level-crossing event must be visible because it is the only one that has been considered as correct. For the same reason, new data should not be acquired during the computation of a valid snapshot.

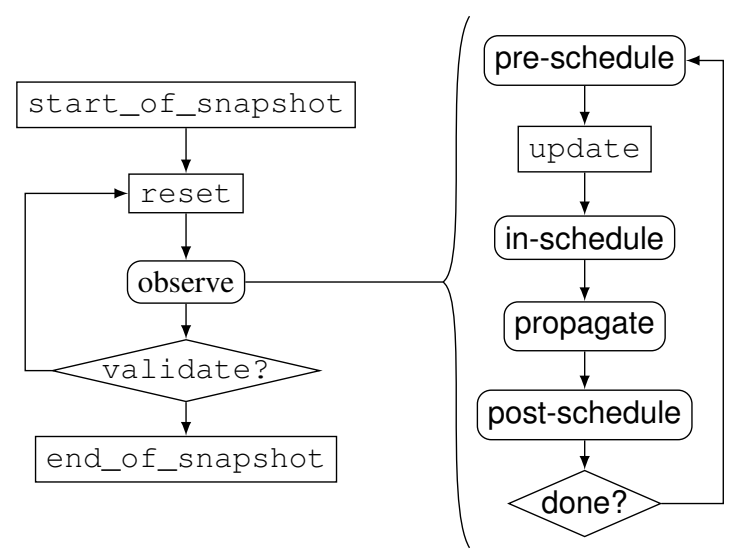

Figure 2: Steps of the generic execution model

\subsection{Discussion on steps}

The overall structure of our execution model is shown on figure 2, with "actor operations" in rectangular boxes, "model of computation operations" in rounded corners boxes, and control choices in diamond shaped boxes.

The schedule operations of the model of computation determine which actors should be observed. These operations are executed each time new data becomes available: at the beginning of an observation (pre-schedule), in order to compute control from the inputs of the model and to determine which actors may produce observable outputs; after update (inschedule) in order to handle data made available on the output ports of the actors; and at the end of the observation (post-schedule), in order to handle the outputs of the model.

Every model of computation must implement preschedule because this operation tells which actors will be observed in the current turn of the loop. The other two schedule operations may do nothing in models of computation where the control does not depend on data.

The first and last steps of the computation of a snapshot insulate the environment of the model from the internal changes that occur in the model during the computation of the observation of its ports. They also insulate the behavior of the actors of a model from the details of the computation of a snapshot of this model, since an actor is not allowed to update its internal state before the end_of_snapshot step. For instance, an actor should not count the number of times its reset, update or validate methods are invoked and make its future behavior depend on this count. 
We can consider the start_of_snapshot step as "sample the external world", and the end_of_snapshot as "update state, act on external world". Between these two steps, actors must have a combinational behavior. This model is therefore very close to the synchronous sequential model where registers are loaded on the ticks of a clock with the results of combinational computations. In our execution model, the clock is the series of instants at which a snapshot exists. We do not need a more elaborate model of time at this level of the execution of a model of a system.

The reset - validate loop is crucial for heterogeneous modeling because it is the way by which the model of computation that is used for the internal model of an actor can influence the model of computation in which the actor is used. In a model of a system, actors are just observed, and the observations are combined by a model of computation to build an observation of the model. However, the behavior of each actor can also be described by a model of the actor (the actor is considered as a subsystem), and the model of computation used to model the behavior of the actor can be different from the first model of computation. We call "external model of computation" the model of computation that is used to combine the behavior of an actor with the behavior of other actors, and "internal model of computation" the model of computation that is used to describe the internal behavior of an actor.

In order to avoid to compute all the possible combinations of models of computation, we hide the internal model of computation to the eyes of the external one. Since the external model of computation "decides" when the ports of an actor are observed, the internal model of computation would have no control on the computation of the snapshot if it could not refuse a computation by making the actor return false to the validate request.

The "observe" loop implements a wellknown technique to compute the behavior of a model as a fixed-point. It is implemented in Ptolemy II by the prefire, fire and postfire methods. prefire is the equivalent of start_of_snapshot in our execution model, fire is equivalent to update and postfire to end_of_snapshot. However, we chose different names since there is no validate - reset loop in the general execution model of Ptolemy II (even if such a validation steps exists in the "Continuous Time" model of computation), and the names of these methods denote the activation of a behavior. Our execution model deals only with observations and we do not limit the behavior of actors to the body of a fire method.

\subsection{Allowing Heterogeneity}

The execution model we have just presented here uses only one model of computation, so one may wonder how heterogeneous models are handled. Our approach of heterogeneity is the same as the hierarchical approach used in Ptolemy (Eker et al., 2002), and our execution model does not depend on the models of computation used to compute the behavior of the actors of a model. It is therefore possible to define the behavior of actors using internal models of computation that differ from their external model of computation.

An issue still subsists: how data produced according to the internal model of computation of an actor will be interpreted in the context of its external model of computation? The behavior of an actor may be expressed using properties that have no meaning in the external model of computation. For instance, an actor may produce time-stamped data samples because its behavior is defined using a timed model of computation, and these samples may be read in a model of computation that has no notion of time. In this case, the series of timed-stamped data samples can be viewed as a sequence of data samples just by discarding the time-stamps, but in the reverse case, when data with no time-stamp is produced in a timed model of computation, a time-stamp must be created for each data sample, and this requires additional information.

Our position is that there is no automatic way to convert data (or control) from a model of computation to another. Often, there are standard ways of adapting the semantics of two models of computation (for instance, periodic sampling can be used to go from continuous time to synchronous data-flow), but such transformations should not be "hard-coded" in the modeling framework nor applied implicitly to an heterogeneous model. The reasons for this are:

- implicit transformations are framework-dependent. This means that the same model could adopt different behaviors when executed in different modeling frameworks;

- several transformations between two models of computation may exist (for instance, when going from discrete to continuous time, it is possible to hold the last value, or to use linear or more complex interpolation). The choice of a transformation is part of the design of the system, and it should therefore appear explicitly in the model;

- even when there is only one possible transformation between two models of computation, using this transformation and setting its parameters is a design choice, and it should appear in the model of the system, with the same importance as the models of computations. 
The main problem with such transformations is that if they are implemented as actors, these actors appear either in the internal or in the external model of computation. Both ways are wrong since they break modularity: if the internal model of an actor contains actors to adapt data to its external model of computation, this internal model depends on the external model of computation. This means that the design of an actor depends on the context in which it will be used. The same problem occurs when the adapting actors are placed in the external model of computation. In (Feredj et al., 2004), we presented a model for domain-polymorph components that allows the adaptation between two models of computation to be done at the interface between the models. This approach turns the adaptation between the semantics of the internal and external models of computation into a property of the edge of the actor.

A last issue is that it is sometimes necessary to define actors which obey several models of computation. For instance, a sampler has a continuous input, a discrete event input (the sampling clock) and a data-flow output (the sequence of samples). A levelcrossing detector has a continuous or sampled input and a discrete event output. Such actors cannot be handled directly in our execution model because only one model of computation is allowed in the model of a system. However, we have shown in (Boulanger et al., 2004) that a flat heterogeneous model, i.e. a model that uses several models of computation at the same level of its hierarchy, can be rewritten automatically into a hierarchical model by projecting heterogeneous actors on the models of computation they use. One may also consider that such behaviors should not be modeled as actors but as transformations between models of computation, and considered as properties of the edge of models, as evoked earlier.

\section{CONCLUSION}

We have presented the roles of a model of computation and the different kinds of actors it should be able to manage, and then an execution model which, by making as few assumptions as possible about actors, is able to execute models that obey any model of computation. Our works on the integration of the reactive synchronous approach into object-oriented programming and on the adaptation between models of computation in the Ptolemy framework make us quite confident in the universality of this model. By considering only observations on the ports of actors, and not the activity of actors, this execution model can safely ignore what happens at lower levels of the hierarchy of a model. This allows the use of different models of computation at different level of the hierarchy of a model of a system. Moreover, by allowing an actor to veto the result of the computation of a snapshot of the model, this execution model allows inner models of computation to interact with the outer models, in addition to the usual control that the outer model has on the inner models of computation.

This execution model requires that a model of computation is able to provide a schedule of the actors of a model, and to propagate the data observed on the output ports toward the input ports. These two operations can be complex, and are, for the moment, implemented using generic programming languages like Java or $\mathrm{C}++$. Our goal is to describe them formally using either an extended version of the Object Constraint Language (OCL) or the Action Language of UML 2, with a mathematical foundation for the in order, particularly, to define transformations from a model of computation to another and to handle heterogeneity in a more generic way.

\section{REFERENCES}

Boulanger, F., Mbobi, M., and Feredj, M. (2004). Flat heterogeneous modeling. In IPSI 2004 conference, http://wwwsi.supelec.fr/fb/download/Articles/IPSI2004.pdf.

Brooks, C., Lee, E. A., Liu, X., Neuendorffer, S., Zhao, Y., and Zheng, H. (2005). Heterogeneous concurrent modeling and design in java (volume 1: Introduction to ptolemy ii). Technical report, University of California, Berkeley.

Eker, J., Janneck, J. W., Lee, E. A., Liu, J., Liu, X., Ludvig, J., Neuendorffer, S., Sachs, S., and Xiong, Y. (2002). Taming heterogeneity the ptolemy approach. In Proceedings of the IEEE, Special Issue on Modeling and Design of Embedded Software.

Feredj, M., Boulanger, F., and Mbobi, M. (2004). An approach for domain polymorph component design. In IEEE International Conference on Information Reuse and Integration 2004 (IRI 2004), http://wwwsi.supelec.fr/fb/download/Articles/IRI2004CDP.pdf.

Fleurey, F., Drey, Z., and Vojtisek, D. (2006). KerMeta Manual. http://www.kermeta.org/docs/KerMetaManual.pdf.

Kong, C. and Alexander, P. (2003). The rosetta meta-model framework. In 10th IEEE International Conference and Workshop on the Engineering of Computer-Based Systems (ECBS'03).

Liu, X., Liu, J., Eker, J., and Lee, E. A. (2003). Heterogeneous modeling and design of control systems. In Software-Enabled Control: Information Technology for Dynamical Systems. Wiley-IEEE Press. 